\title{
Modèles animaux d'ostéoporose post-ménopausique
}

\author{
JP Barlet, V Coxam, MJ Davicco, N Gaumet
}

INRA-Theix, métabolisme minéral et ostéogénèse, croissance et métabolismes des herbivores, 63122 Saint-Genès-Champanelle, France

(Reçu le 24 novembre 1993; accepté le 11 mars 1994)

Résumé - L'ostéoporose est une maladie caractérisée par une faible masse osseuse et accompagnée d'une détérioration de l'architecture microscopique osseuse conduisant à une augmentation de la fragilité osseuse et du risque de fracture. Dans l'espèce humaine, la carence œstrogénique consécutive à la ménopause s'accompagne d'une ostéopénie plus ou moins intense. Chez certaines femmes, cette diminution de la masse osseuse est telle que le seuil fracturaire est dépassé au moindre choc. Divers modèles animaux (chiennes, truies, singes ovariectomisées) sont utilisés pour l'étude de l'étiologie, de la prophylaxie et de la thérapeutique de l'ostéoporose. Néanmoins, bien que les fractures osseuses n'apparaissent jamais chez la ratte ovariectomisée, celle-ci est le modèle le plus fréquemment utilisé car les conséquences osseuses du déficit œstrogénique sont très semblables chez elle et chez la femme ménopausée.

œstrogènes / ménopause / ovariectomie / ratte / os

Summary - Animal models for postmenopausal osteoporosis. Osteoporosis is a disease characterized by low bone mass, microarchitectural deterioration of bone tissue leading to enhanced bone fragility, and a consequent increase in fracture risk. In humans estrogen deficiency following menopause is associated with a more or less intense osteopenia. In some subjects, such a decrease in bone mass induces osteoporosis. Several animal models (ovariectomized female dogs, pigs and monkeys) are used to study the etiology, prophylaxis and treatment for osteoporosis. Nevertheless, the changes in bone metabolism following estrogen deficiency are very similar in rats and humans. Thus, the ovariectomized rat is the most frequently used model for such studies. 


\section{INTRODUCTION}

L'intégrité du corps des Mammifères est assurée par divers mécanismes, d'un degré de perfectionnement variable, dont l'activité persiste plus ou moins longtemps selon l'espèce considérée. Chez l'adulte, l'absence de renouvellement ou de régénération des divers systèmes organiques aboutit à la sénescence (Holliday, 1992). Or, depuis quelques années, par suite de l'augmentation de l'espérance de vie, les problèmes liés à la sénescence prennent une importance croissante. Ainsi, du fait de sa fréquence et du coût de son traitement, l'ostéoporose est un problème de santé publique qui ne cesse de s'aggraver. En France, on compte plus de 50000 cas annuels de fractures du col du fémur (Delmas, 1992). Parmi ces patients, $15 \%$ vont décéder dans l'année qui suit la fracture, et la moitié des survivańts garderont un handicap fonctionnel grave et définitif. En outre, plus de $15 \%$ de la population féminine ayant dépassé la soixantaine souffre de tassements vertébraux. Ainsi, actuellement en France, le coût annuel du traitement de l'ostéoporose est de l'ordre de 4 milliards de francs, coût qui in'inclut pas les conséquences fonctionnelles à long terme. D'où l'intérêt porté au syndrome ostéoporotique, en France (Delmas, 1992) comme à l'étranger (Albright et al, 1941 ; Riggs et Melton, 1986 ; Cooper et Melton, 1992).

\section{MASSE OSSEUSE MAXIMALE, OSTÉOPÉNIE PHYSIOLOGIQUE ET OSTÉOPOROSE}

Le squelette, charpente du corps et banque de calcium de l'organisme, est constitué de 2 types d'os : l'os cortical, dense, et l'os trabéculaire, plus aéré et plus léger. Les 2 sont formés d'éléments de base comparables : les lamelles osseuses. Ces 2 types d'os s'associent pour former les pièces consti- tutives du squelette. L'os cortical haversien, plus résistant aux contraintes mécaniques, représente chez l'adulte jeune environ $80 \%$ de la masse osseuse, mais seulement $15 \%$ des surfaces osseuses déployées. L'os trabéculaire ne représente que $20 \%$ de la masse osseuse. Il contribue à la résistance mécanique des épiphyses osseuses et des corps vertébraux. Formant $85 \%$ des surfaces osseuses, il est très actif dans les échanges métaboliques et dans l'homéostasie calcique.

Trotter et Hixon (1974) ont mesuré le poids et la teneur en calcium de 426 squelettes des 2 sexes depuis l'âge fœtal (16 sem) jusqu'à 100 ans. Ils ont montré que le poids du squelette augmente de façon exponentielle chez le fœtus. Cet accroissement se poursuit irrégulièrement au cours de l'enfance et de l'adolescence, avec un gain de poids très rapide entre l'âge de 10 et 20 ans. La masse osseuse maximale (ou pic de masse osseuse) est atteinte aux alentours de la vingt-cinquième année (Ott, 1990). Elle commence à décroître entre 30 et 40 ans, et cette diminution se poursuit au cours des décennies suivantes (Garn et al, 1967 ; Johnston et Slemenda, 1992 ; Matkovic, 1992), faisant apparaître une ostéopénie physiologique. Chez certains individus, cette réduction du capital osseux est telle que le seuil fracturaire est atteint. II correspond à un contenu minéral osseux de l'ordre de $100 \mathrm{mg} / \mathrm{cm}^{3}$ (valeur maximale $180 \mathrm{mg} / \mathrm{cm}^{3}$, atteinte entre 20 et 30 ans dans les 2 sexes) (Renier et Audran, 1989). À ce stade, tout traumatisme, même minime, est susceptible d'entraîner une fracture. Aussi, depuis la conférence qui s'est tenue à Copenhague en octobre 1990, l'ostéoporose est définie comme une maladie caractérisée par une faible masse osseuse et accompagnée d'une détérioration de l'architecture microscopique osseuse conduisant à une augmentation de la fragilité osseuse et du risque de fracture (Bouillon et al, 1991). 
Riggs et Melton (1986) ont montré qu'il existait 2 types d'ostéoporose. L'ostéoporose sénile (ou de type II) survient chez la femme et chez l'homme de plus de 70 ans. Elle se traduit surtout par la fracture du col du fémur. Son étiologie, multifactorielle, fait intervenir une diminution de l'activité ostéoblastique liée à l'âge et un hyperparathyroïdisme secondaire, également d'origine multifactorielle. L'ostéoporose post-ménopausique (ou de type I) survient dans la période de 5 à 15 ans suivant la ménopause. Elle est la conséquence du déficit œstrogénique consécutif à la cessation d'activité ovarienne. La perte osseuse affecte alors essentiellement l'os trabéculaire et se traduit surtout par des tassements vertébraux.

\section{CONSÉQUENCES DU DÉFICIT CESTROGÉNIQUE AU NIVEAU SQUELETTIQUE}

L'existence d'une perte osseuse pendant les 20 années précédant la ménopause est contestée. Elle existe probablement, mais est faible, de l'ordre de 0,2\%-0,3\% par an chez certaines femmes pendant les 5 ans précédant la ménopause (Delmas, 1992). Elle pourrait résulter d'une carence lutéale en progestérone (Prior, 1990) dont les récepteurs sont présents au niveau ostéoblastique (Wei et al, 1993). Heaney et al (1978) ont été les premiers à démontrer que, chez la femme ménopausée, la résorption osseuse l'emportait sur l'accrétion, ce qui entraînait une perte osseuse. De nombreuses études histomorphométriques et/ou biochimiques ont confirmé cette accélération du turnover osseux après la ménopause (Delmas et al, 1983 ; Johnston et al, 1985 ; Riis et al, 1986). Selon Parfitt et al (1983), la phase de perte osseuse rapide consécutive à la ménopause résulterait de l'accélération du turnover osseux et d'une activité ostéoclastique accrue. Puis, après quelques
5 années, le turnover ralentirait et la perte osseuse continuerait, mais à un rythme ralenti, résultant d'une diminution de l'activité ostéoblastique (Arlot et al, 1984). Les résultats de Stepan et al (1987) obtenus chez 12 jeunes femmes ovariectomisées confirment cette hypothèse. Les traitements œestrogéniques substitutifs freinent la résorption (et l'accrétion) et suppriment la perte osseuse chez la femme ménopausée (Christiansen et al, 1982) comme chez la ratte OVX (Wronski et al, 1988b). En outre, chez cette dernière, ils pourraient également stimuler la différenciation et l'activité ostéoblastiques (Chow et al, 1992). Depuis quelques années, on sait que les ostéoblastes possèdent des récepteurs au $17 \beta-$ œstradiol (Eriksen et al, 1988 ; Komm et al, 1988 ; Takano-Yamamoto et Rodan, 1990). L'effet du stéroiide au niveau ostéoblastique peut donc être direct et/ou indirect, faisant intervenir une production accrue d'IGF-1 (Ernst et al, 1989 ; Franchimont, 1989). Cependant, chez la ratte de 2 ans, OVX depuis 1 an, IGF-1 stimule surtout la résorption osseuse (lbbotson et al, 1992).

$\mathrm{Si}$ les œstrogènes ont un rôle préventif vis-à-vis de l'ostéoporose, ils ne possèdent aucun effet curatif, puisqu'ils ne restaurent pas la masse osseuse (Schot et Schuurs, 1990). De plus, ils sont contre-indiqués chez les femmes prédisposées à certaines affections endométriales ou cardiovasculaires (Duursma et al, 1992).

\section{MODĖLES ANIMAUX D'OSTÉOPOROSE POST-MÉNOPAUSIQUE}

\section{Définition d'un modèle animal}

Chez le modèle utilisé pour l'étude d'une pathologie déterminée, celle-ci doit induire des séquelles identiques à celles observées dans l'espèce humaine. Un modèle animal d'ostéoporose post-ménopausique peut 
donc être défini comme un animal vivant chez qui l'on observe une perte osseuse après arrêt spontané ou induit de l'activité ovarienne, et chez lequel les caractéristiques biochimiques et histomorphométriques et les conséquences physiologiques et pathologiques de cette perte sont analogues à celles survenant chez la femme ménopausée.

La ménopause ne survient que chez les primates. Hormis ceux-ci, il n'existe donc aucun modèle animal reproduisant parfaitement les conséquences physiologiques post-ménopausiques. Néanmoins plusieurs espèces sont utilisées, avec plus ou moins de succès, pour les travaux concernant l'étiologie, la prévention et/ou le traitement de l'ostéoporose.

\section{Modèle canin}

Le squelette du chien adulte est analogue au squelette humain en ce qui concerne structure et remodelage. Le rapport os compact/os trabéculaire est respectivement de $4 / 1$ et de $5 / 1$ chez le chien et chez l'homme (Gong et al, 1964 ; Parks et al, 1986). Le remodelage des ostéons s'effectue de façon identique dans les espèces canine et humaine, bien que légèrement plus rapide dans la première (Frost, 1969 ; Kimmel et Jee, 1982).

Selon Malluche et al (1986), chez la chienne Beagle de 5 ans, l'ovariectomie induit une ostéopénie. Celle-ci survient en 2 temps. La première phase débute au cours du mois suivant l'opération et se manifeste essentiellement au niveau trabéculaire. La seconde phase résulte d'une hypoactivité ostéoblastique et peut être corrigée partiellement par administration de 1,25-dihydroxycholécalciférol (Malluche et Faugère, 1990). Par contre, selon Martin et al (1987), l'ovariectomie n'aurait pas d'effet important sur les propriétés mécaniques de la diaphyse et du col du fémur ni sur celles des vertèbres lombaires. L'effet le plus notable de la carence en œstrogènes chez la chienne est certainement l'amincissement du tissu osseux compact au niveau appendiculaire (Karambolova et al, 1987). Néanmoins, l'étude histomorphométrique de Boyce et al (1990) portant sur des biopsies osseuses prélevées sur 25 chiennes à 1, 6 et 10 mois après ablation des ovaires (pratiquée à 18 mois) ne permettait pas de mettre en évidence des modifications importantes de la masse osseuse et concluait au manque d'intérêt de ce modèle, utilisé ici sans témoin pseudo-opéré.

L'absence d'effets très marqués de l'ovariectomie chez la chienne peut sans doute s'expliquer, en partie, par la grande dissemblance des profils hormonaux et de la durée des cycles dans les espèces canine et humaine (Kimmel, 1991a).

\section{Modèle ovin}

Le remodelage osseux de l'espèce ovine est très semblable à celui de l'espèce humaine (Chavassieux et al, 1991a). Aussi, la brebis et l'agneau ont été utilisés pour l'étude du métabolisme osseux après ingestion de fluorure de sodium, expérimenté dans le traitement de l'ostéoporose postménopausique (Chavassieux et al, 1991b). L'ovariectomie semble accélérer le remodelage osseux chez ces animaux (Pastoureau et al, 1989). Néanmoins, chez 7 brebis de 7 ans, 6 mois après castration, la densité osseuse mesurée par absorptiométrie biphotonique au niveau du fémur gauche et des vertèbres lombaires (L1-L2), et par tomodensitométrie quantitative (Pouilles et Ribot, 1992) au niveau du col du fémur et de L1-L3, n'était pas significativement différente de celle mesurée chez 7 témoins pseudo-opérés (tableau 1). Les propriétés mécaniques (résistance à la torsion, souplesse, degré de déformation ultime) n'étaient pas non plus significativement dif- 
Tableau I. Densité osseuse mesurée par absorptiométrie biphotonique (DPA) ou par tomodensitométrie quantitative (QCT) chez la brebis de 7 ans pseudo-opérée (témoin) ou ovariectomisée (OVX) depuis 6 mois.

\begin{tabular}{lcc}
\hline Densité osseuse & Témoins & OVX \\
\hline DPA $\left(g / \mathrm{cm}^{2}\right)$ & & \\
$\quad$ Fémur total & & \\
$\quad$ Epiphyse fémorale proximale & $1,028 \pm 0,033$ & $0,937 \pm 0,059$ \\
$2^{\mathrm{e}}$ vertèbre lombaire & $0,779 \pm 0,075$ & $0,741 \pm 0,014$ \\
& $0,936 \pm 0,038$ & $0,848 \pm 0,043$ \\
QCT (mg/cm $\left.{ }^{3}\right)$ & & \\
$\quad$ Col du fémur & $458 \pm 14$ & $302 \pm 13$ \\
$\quad$ Vertèbres lombaires & $350 \pm 21$ & \\
$\quad($ L1-L2-L3) & & \\
\hline
\end{tabular}

Moyenne \pm écart type ; 7 animaux dans chaque groupe (Geusens, Caulin et Barlet, données non publiées).

férentes chez les OVX et chez les témoins (tableau II).

Pourquoi le squelette de la brebis est-il peu influencé par la castration ? La brebis est herbivore: son régime est parfois très riche en calcium. Ainsi, par exemple, une brebis consommant quotidiennement $3 \mathrm{~kg}$ de foin de luzerne ingère simultanément plus de $50 \mathrm{~g}$ de calcium ! Mais l'absence d'effets marqués de l'ovariectomie sur le squelette ovin peut sans doute s'expliquer en grande partie par le fait que, chez les bovins et les ovins, en dehors de la période préovulatoire (fig 1), la contribution ovarienne au maintien à niveau de l'œstradiolémie est très faible. L'essentiel est dû à l'activité corticale surrénalienne ou à l'aromatisation périphérique. En outre, chez ces animaux, les concentrations plasmatiques en $17 \alpha$-oestradiol sont 3 à 4 fois supérieures à celles en $17 \beta$-oestradiol (Saumande, communication personnelle). In vivo, les effets du $17 \alpha$-œstradiol perfusé à la ratte OVX, évalués par histomorphométrie de l'os tra-

Tableau II. Propriétés mécaniques du fémur gauche ou de la première vertèbre lombaire (L1) de la brebis de 7 ans pseudo-opérée (témoin) ou ovariectomisée (OVX) depuis 6 mois.

\begin{tabular}{lcc}
\hline & Témoins & OVX \\
\hline Angle de déformation maximale du fémur entier (degrés) & $11,8 \pm 0,7$ & $12,6 \pm 0,5$ \\
Tension maximale $\left(10^{6} \mathrm{~N} / \mathrm{m}^{2}\right)^{*}$ & & \\
Col du fémur & $3,150 \pm 0,337$ & $2,507 \pm 0,214$ \\
L1 & $2,797 \pm 0,298$ & $2,350 \pm 0,253$ \\
\hline
\end{tabular}

Moyenne \pm écart type ; 7 animaux dans chaque groupe (Geusens, Caulin et Barlet données non publiées) ;

* mesure effectuée sur une éprouvette osseuse. 

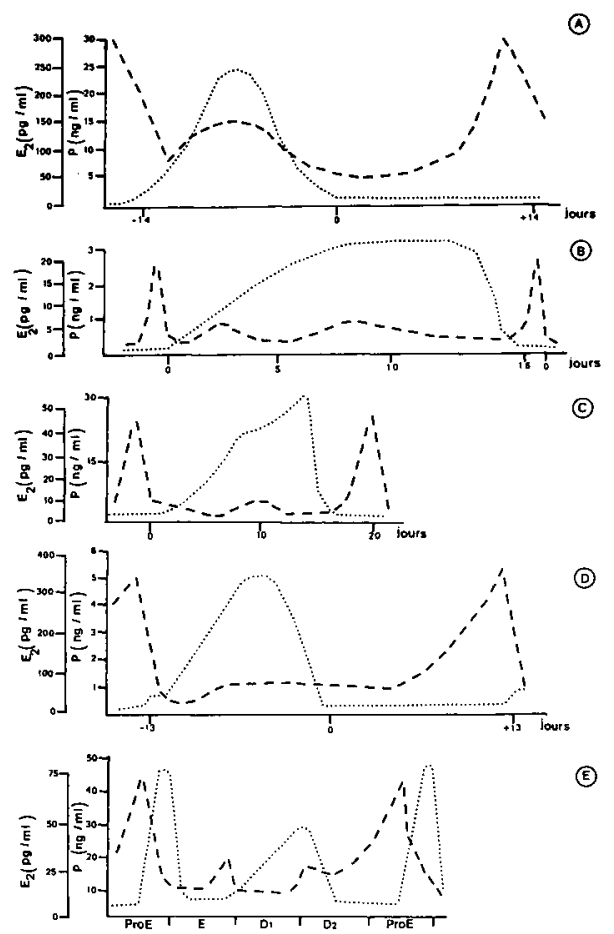

(E)

Fig 1. Profils hormonaux au cours du cycle oestral chez la femme (A), la brebis $(B)$, la truie (C), le macaque femelle (D) et la ratte $(E)\left(E_{2}\right.$ : œstradiolémie (tirés) ; $P$ : progestéronémie (pointillés) (d'après Driancourt et al, 1991).

béculaire, ne dépasseraient pas le $1 / 50$ de ceux du 17 $\beta$-oestradiol (Takano-Yamamoto et Rodan, 1990).

\section{Modèle porcin}

Deux types de porcs sont utilisés en recherche biomédicale : le porc normal et le miniporc, dont le poids adulte ne dépasse pas $60 \mathrm{~kg}$, la race Pot-Bellied originaire de I'Asie du Sud-Est étant la plus petite. Comme l'espèce humaine, l'espèce porcine est omnivore. En outre, la truie présente des cycles cestriens d'une durée de 18-21 j, continus au cours d'une année (fig 1). De plus, si l'os trabéculaire porcin est plus dense que celui de l'homme, les remodelages de celui-ci et de l'os cortical sont identiques chez les 2 espèces (Mosekilde et al, 1987). C'est ce qui explique sans doute l'utilisation de truies adultes normales, dont le poids dépassait $230 \mathrm{~kg}$, pour l'étude des effets de l'intensité et de la durée d'un exercice physique (marche sur un tapis roulant à plus ou moins grande vitesse) sur l'histomorphométrie des os porteurs (fémurs) (Raab et al, 1991) ou non (côtes) (Tommerup et al, 1993). Chez la minitruie Sinclair S-1 de 10 mois, OVX à l'âge de 4 mois et dont la teneur en calcium du régime a été diminuée de $0,9 \%$ à $0,75 \%$, la densité minérale osseuse et le volume d'os trabéculaire diminuent respectivement de $6 \%$ et $15 \%$. Cet animal peut donc être un modèle intéressant (Mosekilde et al, 1993).

\section{Modèle simien}

Les primates sont les animaux les plus proches de l'espèce humaine. Beaucoup d'entre eux ayant une posture verticale, les forces mécaniques auxquelles sont soumis leurs os sont identiques à celles auxquelles est soumis le squelette humain. Les cycles menstruels du macaque femelle ressemblent beaucoup à ceux de la femme (fig t). De plus, entre 20 et 30 ans, ces animaux présentent des cycles irréguliers ou une aménorrhée, une élévation des gonadotrophines circulantes associée à une faible œstradiolémie et progestéronémie, tout comme au début de la ménopause humaine (Hodgen et al, 1977). La densité osseuse mesurée au niveau de l'humérus et des vertèbres diminue chez les femelles de plus de 30 ans (Pope et al, 1989) et la castration chirurgicale (Miller et al, 1986) ou chimique (Mann et al, 1990) entraîne une dimi- 
nution de la masse osseuse trabéculaire et de la densité de l'os cortical (Lundon et Grynpas, 1993). Cependant, chez des Babouins capturés à l'état sauvage et maintenus en captivité, bien que le réseau trabéculaire de la crête iliaque soit plus compact que celui observé dans l'espèce humaine, le taux de formation osseuse $y$ est inférieur (Schnitzler et al, 1993). Une perte osseuse consécutive à l'ovariectomie a également été rapportée chez ces animaux (Jérome et al, 1986) et chez les Rhésus (Longscope et al, 1989). Chez les premiers, 6 ou 12 mois après ovariectomie, l'activité phosphatase alcaline plasmatique et l'ostéocalcinémie (marqueurs de l'activité ostéoblastique) sont élevées, tout comme l'est l'activité phosphatase acide résistante au tartrate (marqueur de l'activité ostéoclastique), alors que la densité des vertèbres lombaires (mesurée par absorptiométrie biphotonique) est diminuée (Thompson et al, 1992). Cependant, si l'utilisation des modèles canin, ovin, porcin ou murin ne pose pas de problèmes financiers ou juridiques particuliers, le principal obstacle à l'utilisation plus fréquente des singes dans les travaux sur l'ostéoporose est sans doute lié au prix très élevé de ces animaux et, surtout, à la législation draconienne réglementant leurs conditions d'élevage.

\section{Modèle murin}

La souris et le rat sont utilisés comme modèles d'ostéoporose humaine.

\section{La souris}

L'ostéoporose humaine, postménopausique ou sénile, est d'origine multifactorielle. La masse osseuse maximale est déterminée par divers facteurs, dont l'hérédité (Pocock et al, 1987). C'est la raison pour laquelle, chez la souris, la souche SAM (Senescence Accelerated Mouse), caractérisée par une très faible masse osseuse maximale à l'âge adulte, est considérée comme un bon modèle d'ostéoporose sénile (Tsuboyama et al, 1993). Une masse osseuse aussi taible résulterait d'une résorption accélérée (Kawase et al, 1989), associée à un anabolisme ralenti pendant le remodelage de l'os cortical (Tsuboyama et al, 1993). En outre, chez la souris, l'ovariectomie entraîne une perte osseuse résultant de la prolifération des ostéoclastes, induite par l'élévation des concentrations plasmatiques d'interleukine- 6 consécutive à la carence cestrogénique (Jilka et al, 1992).

\section{Le rat}

Si le rat mâle âgé peut également être choisi comme modèle d'ostéoporose sénile (Vanderschueren et al, 1993), c'est la ratte OVX qui est l'un des modèles d'ostéoporose postménopausique les plus utilisés. Contrairement à I'homme chez qui la croissance squelettique cesse dès la maturité et chez qui la diminution de la masse osseuse débute aux alentours de la vingt-cinquième année pour se poursuivre tout au long de la vie, chez le rat, la croissance des os est continuelle (Donaldson, 1919). Chez la femme d'une cinquantaine d'années, le processus d'ossification épiphysaire a cessé depuis la maturité, l'ossification endochondrale ne se poursuit que très lentement, et le remodelage osseux est le principal processus grâce auquel le squelette maintient une certaine intégrité (Frost, 1985). Le modèle animal le mieux approprié à l'étude de l'ostéoporose serait donc un animal âgé, chez qui la croissance en longueur aurait cessé, mais avec un remodelage osseux persistant.

La ratte n'est certainement pas un modèle parfait pour l'ostéoporose postménopausique:

- la durée du cycle œstrien murin est différente de celle du cycle menstruel feminin, tout comme le sont les profils hormonaux 
chez ces 2 espèces (fig 1 ). Les cycles œstriens deviennent irréguliers vers l'âge de 10 mois, pour cesser vers 20 mois. Mais une certaine activité ovarienne persiste, même chez la ratte très âgée, comme en témoigne la diminution du poids utérin observée après ovariectomie des rattes de 30 mois (tableau III).

- La masse osseuse de la ratte alimentée convenablement ne diminue pas au cours du vieillissement (Hansard et Crowder, 1957). Au contraire, le contenu minéral osseux (mesuré par absorptiométrie biphotonique) des vertèbres lombaires augmente entre 2 et 32 mois chez certaines lignées de rattes Wistar (Juhn et al, 1991).

- La plupart des cartilages de conjugaison des os longs des rats mâles Wistar ne sont pas soudés avant l'âge de 30 mois (Kimmel, 1991b), bien que cette fermeture puisse être plus précoce chez les femelles de même souche (Joss et al, 1963).

- Les rongeurs sont dépourvus d'os lamellaire (Draper, 1985), et le remodelage n'existe pas dans l'os cortical du rat, démuni d'ostéons haversiens (Frost, 1973 ; Kalu, 1984, 1971).

- Le remodelage, lorsqu'il existe chez le rat, est différent de celui observé chez l'homme, chez qui la formation osseuse s'effectue sur le lieu même de la résorption. Chez le rat en croissance, la plus grande partie de l'os spongieux se forme sur des surfaces non résorbées. Ce qui indique que, chez le jeune rat, les 2 processus ne sont pas liés par voie paracrine, mais par d'autres signaux, tels que des stimulations mécaniques (Chow et al, 1993).

- L'ovariectomie n'induit jamais l'apparition de fractures chez la ratte, même très âgée (Kalu et al, 1989) ou carencée en calcium (Hodgkinson et al, 1978).

Comment alors expliquer l'utilisation aussi fréquente du rat dans les travaux concernant l'ostéoporose ? II importe tout d'abord de distinguer perte osseuse post-ménopausique et ostéoporose post-ménopausique. Si une perte osseuse survient effectivement chez toutes les femmes après la ménopause, toutes, heureusement, ne souffrent pas du syndrome ostéoporotique. $\mathrm{Ce}$ qui démontre que des facteurs de risque autres que la cessation d'activité ovarienne contribuent à l'étiologie de l'ostéoporose. Ainsi, un apport alimentaire calcique insuffisant et/ou une mauvaise absorption calcique intestinale seraient des facteurs très importants dans l'étiologie de l'ostéoporose

Tableau III. Poids vif $(\mathrm{g})$ et poids de l'utérus $(\mathrm{mg})$ chez des rattes.
Wistar (6 mois)
Poids vif $\quad$ Poids utérus
Lou (30 mois)
Poids vif $\quad$ Poids utérus

$\begin{array}{lll}\text { SH } & & \\ \text { OVX } & 295 \pm 15 & 759 \pm 87 \\ \text { E } & 281 \pm 10 & 174 \pm 27^{\star \star} \\ \text { P } & 299 \pm 11 & 823 \pm 68 \\ E+P & 293 \pm 7 & 269 \pm 82^{\star \star} \\ & 298 \pm 13 & 807 \pm 91\end{array}$

\footnotetext{
Rattes de souche Wistar de 6 mois ou de souche Lou de 30 mois, un mois après ovariectomie (OVX) seule, OVX et traitement au $17 \beta$-estradiol (E; $10 \mu \mathrm{g} / \mathrm{kg} P V / 48 \mathrm{~h}, \mathrm{sc}$ ), OVX et progestérone ( $\mathrm{P} ; 140 \mu \mathrm{g} / \mathrm{kg} P V / 48 \mathrm{~h}, \mathrm{sC}), \mathrm{OVX}$ et $E+P$ (mêmes doses) ou pseudo-ovariectomisées $(\mathrm{SH})(n=10)$ pour chaque lot ; moyenne \pm écart type ; $p<$ $0,05,{ }^{* *} p<0,01$, comparaison par rapport au lot $\mathrm{SH}$ ) (Gaumet, données non publiées).
} 
(Heaney, 1982 ; Nordin et al, 1985 ; Riggs et al, 1987). En effet, l'ostéopénie physiologique axiale et appendiculaire liée à la ménopause peut être considérablement ralentie et diminuée lorsque l'ingestion journalière de calcium par des femmes ménopausées passe de moins de $400 \mathrm{mg}$ à 1000 mg (Reid et al, 1993). De plus, la densité minérale osseuse prépubertaire est accrue lorsque l'ingestion calcique est augmentée au cours de l'enfance (Johnston et al, 1992). Or, à l'exception d'une expérimentation réalisée chez le lapin, chez qui le niveau d'ingestion calcique n'a pas d'effet significatif sur la masse osseuse maximale adulte (Gilsanz et al, 1991), à notre connaissance, seulement 3 études concernant les effets du régime alimentaire sur l'ostéopénie consécutive à la carence œstrogénique ont été réalisées chez la ratte.

Dans la première expérimentation (Izawa et al, 1985) la supplémentation des animaux en analogues de la vitamine $D$ inhibait la perte osseuse consécutive à l'ovariectomie en augmentant l'absorption calcique intestinale. Dans la seconde (Tabuchi et al, 1986), l'administration de dihydrotachysterol à des rattes OVX prévenait la perte d'os cortical en stimulant la prolifération des précurseurs mésenchymateux des ostéoblastes. Dans la dernière (Lazowski et al, 1991), une supplémentation calcique distribuée pendant 6 sem avant et après ovariectomie préservait la masse osseuse selon un mécanisme indéterminé.

Cependant, chez la ratte, en l'absence d'une restriction alimentaire, la prise de poids entraînée par la boulimie induite par la castration risque de perturber l'ostéopénie induite par la carence œstrogénique (Wronski et al, 1986a). Néanmoins, chez de jeunes rattes OVX à l'âge de 1 mois et recevant quotidiennement la même quantité d'aliment que celle consommée par les témoins pseudo-opérés, un exercice physique (course sur tapis roulant à la vitesse maximale de $15-20 \mathrm{~m} / \mathrm{min}$ pendant $60 \mathrm{~min}$,
5 j par semaine pendant $8 \mathrm{sem}$ ) diminuait la résorption osseuse basale ou induite par l'ovariectomie et augmentait la masse osseuse fémorale et vertébrale. Le même exercice physique pratiqué par des rattes OVX à 9 mois n'avait pas d'effet marqué sur leur squelette (Yeh et al, 1991). De même, chez la femme, l'effet bénéfique au niveau du squelette d'un exercice physique modéré est bien marqué au cours de la jeunesse alors qu'il l'est très peu lorsqu'une activité analogue commence à être pratiquée seulement après la ménopause (Eisman et al, 1991). Ainsi la ratte est un très bon modèle d'ostéopénie induite par carence œstrogénique.

Le squelette du rat subit des modifications importantes au cours de la vie de l'animal (Reisenfeld, 1981 ; Simon, 1984 ; Schapira et al, 1991), et celles-ci diffèrent quantitativement selon le sexe de l'animal (Mbuyi-Muamba et Dequecker, 1986 ; Kiebzack et al, 1988). Pendant les premiers mois post-nataux survient une ostéogénèse rapide (Nishunoto et al, 1985). Ce processus se poursuit pendant la première année de vie (Bar-Shira-Maymon et al, 1989). Après l'apparition de la masse osseuse maximale, celle-ci diminue en fonction de l'âge, diminution visible au niveau des os axiaux et appendiculaires (Kalu, 1984 ; Silbermann et al, 1989 ; Li et al, 1991). C'est Saville (1969) qui, le premier, rapporta l'apparition d'une ostéopénie chez les rattes OVX. De nombreux travaux ont confirmé ce résultat (Aitken et al, 1972 ; Lindgren et Lindholm, 1979 ; Bealt et al, 1984 ; Pohlman et al, 1985 ; Faugère et al, 1986 ; Tabuchi et al, 1986 ; Turner et al, 1987 ; Hock et al, 1988 ; Hori et al, 1988 ; Wronski et al, 1989a ; Kimmel et Wronski, 1990). Bien que cette perte osseuse consécutive à la castration soit visible au niveau cortical (Kalu, 1984 ; Faugère et al, 1986 ; Tabuchi et al, 1986), elle est beaucoup plus prononcée au niveau trabéculaire (Kimmel et Wronski, 1990). Les études basées sur des mesures physiques 
(Cruess et Hong, 1979), biochimiques ou histomorphométriques (Turner et al, 1987 ; Kalu et al, 1988 ; Wronski et al, 1985, 1986b, 1988a, 1988b, 1989c) démontrent que l'ovariectomie stimule le turnover à la fois au niveau cortical et trabéculaire. Une augmentation transitoire de la croissance osseuse longitudinale survient également chez les rattes OVX, mais celle-là redevient normale au plus tard 2 mois après l'opération (Wronski et al, 1988a). La séquence concernant l'augmentation du turnover osseux et la perte d'os trabéculaire est biphasique, comme chez la femme ménopausée (Wronski et al, 1989c). La phase initiale de perte osseuse rapide coïncide avec l'augmentation maximale du turnover osseux. Cette phase initiale est suivie d'une période intermédiaire au cours de laquelle le turnover se ralentit et le volume d'os trabéculaire se stabilise à un niveau ostéopénique. Survient une dernière phase de perte osseuse latente associée à un turnover osseux accéléré. Comme chez la femme ménopausée, la perte importante d'os trabéculaire observée chez la ratte OVX ne peut avoir lieu que si l'augmentation de la résorption est plus importante que celle de la formation (Wronski et Yen, 1991). Bien que la plupart des études histomorphométriques aient été effectuées au niveau du tibia, l'ostéopénie et l'augmentation du turnover osseux sont également visibles au niveau vertébral (Wronski et al, 1986b). Cette ostéoponie trabéculaire vertébrale se développe cependant plus lentement et est moins prononcée à ce niveau qu'à celui du tibia (Wronski et al, 1989a). Plusieurs études ont démontré que, chez la ratte OVX, un traitement œstrogénique inhibait l'accélération du turnover osseux et protégeait efficacement l'animal contre l'ostéopénie (Turner et al, 1987 ; Wronski et al, 1988b) comme le font également les bisphosphonates (Wronski et al, 1989b). CEstrogènes et bisphosphonates ont donc le même effet préventif chez la ratte $O V X$ et chez la femme ménopausée (Wronski et Yen, 1991).
Enfin, au cours de la carence cestrogénique, la réponse de la ratte à l'effet anabolisant de l'hormone parathyroïdienne (PTH) est analogue à celle observée chez la femme. In vitro, l'effet anabolisant de PTH au niveau osseux fait intervenir essentiellement une production ostéoblastique accrue d'IGF-I (Canalis et al, 1989). In vivo, chez la ratte âgée (14-16 mois), cet effet anabolisant de PTH (4-16 $\mu \mathrm{g} / 100 \mathrm{~g} / \mathrm{j}$, pendant $12 \mathrm{j}$ ) est très variable (Hock et Wood, 1991), comme il l'est chez la femme âgée ostéoporotique (Reeve et al, 1980). Cette diminution liée à l'âge de la réponse osseuse à l'effet anabolisant de PTH peut résulter d'une diminution simultanée de la stimulation de l'absorption calcique intestinale, résultant elle-même d'une moindre élévation des concentrations plasmatiques de 1,25-dihydroxycholécalciférol (Hock et Wood, 1991) et d'une diminution des récepteurs entérocytaires au stérö̈de au cours du vieillissement (Horst et al, 1990). L'inconsistance de la réponse de l'anabolisme osseux à PTH au cours du vieillissement pourrait aussi résulter d'une diminution des cellules-souches des ostéoblastes, déjà mise en évidence in vitro (Eriksen et al, 1985). II a en outre été récemment démontré que, chez la ratte $O V X$ à l'âge de 16 sem, un traitement associant de la $21^{e}$ à la $25^{\ominus}$ semaine $17 \beta$-oestradiol $(50 \mu \mathrm{g} / \mathrm{kg} / \mathrm{j})$ et fragment (1-34) de la PTH humaine $(2,5 \mu \mathrm{g} / \mathrm{kg} / \mathrm{j})$ diminuait la résorption osseuse et maintenait un anabolisme osseux intense. Néanmoins le volume d'os trabéculaire du tibia proximal des animaux traités, bien que très supérieur à celui des témoins ovariectomisés, demeurait inférieur à celui mesuré chez les pseudo-opérés, par suite d'un nombre plus faible de trabécules, lesquels étaient également plus minces (Shen et al, 1992). À notre connaissance, jusqu'à présent, l'association PTH-œestradiol n'a encore jamais été utilisée dans la prévention ou le traitement de l'ostéoporose postménopausique. 
En conclusion, il n'existe aucun modèle animal parfait d'ostéoporose post-ménopausique ou sénile. Chacun de ceux les plus fréquemment utilisés présente quelques avantages pour approfondir certains aspects de cette pathologie multifactorielle. Les fractures osseuses n'apparaissent jamais chez la ratte jeune ou âgée, même après ablation des ovaires, peut-être parce que les forces mécaniques s'exerçant sur le squelette d'un quadrupède ou d'un bipède sont différentes. Néanmoins la similitudes des perturbations du métabolisme osseux observées chez la femme et chez la ratte au cours de la carence œstrogénique, spontanée (ménopause) chez la première ou induite (ovariectomie) chez la seconde, prouve que la ratte OVX est un bon modèle pour l'étude de l'étiologie, de la prophylaxie et de la thérapeutique de l'ostéoporose post-ménopausique humaine. En effet, pendant celle$\mathrm{ci}$, il se produit une diminution de la masse osseuse, accompagnée de modifications de l'architecture de l'os trabéculaire, qui aboutissent finalement à des altérations des propriétés biomécaniques, correspondant à une augmentation de la fragilité osseuse, caractéristique fondamentale de l'ostéoporose.

\section{RÉFÉRENCES}

Aitken JM, Armstrong B, Anderson JB (1972) Osteoporosis after oophorectomy in the mature female rat and the effect of estrogen or progestogen replacement therapy in its prevention. J Endocrinol 55, 79-87

Albright F, Smith PH, Richardson AM (1941) Postmenopausal osteoporosis: its clinical features. $J$ Am Med Ass 116, 2465-2474

Arlot M, Edouard C, Meunier PJ, Neer RM, Reeve $\mathrm{J}$ (1984) Impaired osteoblast function in osteoporosis. Comparison between calcium balance and dynamic histomorphometry. Brit Med J 289, 517-520

Bar-Shira-Maymon B, Coleman R, Cohen A, Steinhagen-Thiessen E, Silbermann M (1989) Age-related bone loss in lumbar vertebrae of
CW-1 female mice: a histomorphometric study. Calcif Tissue Int 36, 123-125

Bealt PT, Misra LK, Young RL, Spjut HJ, Evans $\mathrm{HJ}$, Leblanc A (1984) Clomiphene protects against osteoporosis in the mature ovariectomized rat. Calcif Tissue Int 36, 123-125

Bouillon $\mathrm{R}$, Burckhardt $\mathrm{P}$, Christiansen $\mathrm{C}$ et al (1991) Consensus Development Conference: prophylaxis and treatment of osteoporosis. Osteoporosis Int 1, 114-117

Boyce RW, Francks AF, Jankowsky ML, Orcutt CM, Piacquadio AM, White JM, Bevan JM (1990) Sequential histomorphometric changes in cancellous bone from ovariohysterectomized dogs. J Bone Miner Res 5, 947-953

Canalis E, Centrella M, Burch W, Mc Carthy TL (1989) Insulin-like growth factor I mediates selective anabolic effects of parathyroid hormone in bone cultures. J Clin Invest 83, 60-65

Chavassieux P, Pastoureau P, Boivin G, Chapuy MC, Delmas PD, Meunier PJ (1991a) Dose effect on ewe bone remodeling of shortterm sodium fluoride administration. A histomorphometric and biological study. Bone 12, 421-427

Chavassieux $P$, Pastoureau $P$, Boivin G, Chapuy MC, Delmas $P D$, Milhaud G, Meunier PJ (1991b) Fluoride-induced bone changes in lambs during and after exposure to sodium fluoride. Osteoporosis Int 2, 26-33

Chow J, Tobias $\mathrm{JH}$, Colston KW, Chambers TJ (1992) Estrogen maintains trabecular bone volume in rats not only by suppression of bone resorption but also by stimulation of bone formation. J Clin Invest 89, 74-78

Chow JWM, Badve S, Chambers TJ (1993) A comparison of microanatomic basis for coupling between bone formation and bone resorption in the rat. Bone 14, 355-360

Cooper C, Melton LJ (1992) Epidemiology of osteoporosis. Trends Endocrinol Metab 3, 224-229

Christiansen C, Christensen MS, Larsen NE, Transbol IE (1982) Pathophysiological mechanisms of estrogen effect on bone metabolism. Dose-response relationships in early postmenopausal women. J Clin Endocrinol Metab 55, 1124-1130

Cruess RL, Hong KC (1979) The effect of longterm estrogen administration on bone metabolism in the female rat. Endocrinology 104, 1188-1192 
Delmas PD (1992) Bases physiologiques des ostéoporoses. In : Ostéoporose : pour une prévention nutritionnelle du risque (B Lesourd, CH Rapin, P Sachet, eds). Cerin, Paris, 5-12

Delmas PD, Wahner HW, Mann KG, Riggs BL (1983) Assessment of bone turnover in postmenopausal osteoporosis by measurement of serum bone Gla-protein. J Lab Clin Med $102,470-476$

Donaldson HH (1919) Quantitative studies on the growth of the skeleton of the albino rat. $A m J$ Anat 26, 237-314

Draper HH (1985) Osteoporosis: animal models for the study of nutrition and disease. $I n$ : Advances in Nutrition Research (RP Berd, NJH Mercer, HH Draper, eds). Plenum Press, New York, 172-186

Driancourt MA, Royère $D$, Héron $B$, Levasseur MC (1991) Cycles oestriens et cycles menstruels. In : La reproduction chez les mammifères et chez l'homme (C Thibault, MC Levasseur, eds). Édition Marketing, Paris, 573-581

Duursma SA, Raymakers JA, Boereboom FTJ, Scheven RAA (1992) Estrogen and bone metabolism. Obstet Gynecol Survey 47, 38-44

Eisman JA, Kelly P, Pocock N, Sambrook PN (1991) Exercise and bone density. Osteoporosis Int 1, 118-119

Eriksen EF, Mosekilde L, Melsen F (1985) Trabecular bone resorption depth decreases with age: differences between normal males and females. Bone 6, 141-146

Eriksen EF, Colvard DS, Berg NJ, Graham ML, Mann KG, Spelsberg TC, Riggs BL (1988) Evidence of estrogen receptors in normal human osteoblast-like cells. Science 241, 84 86

Ernst M, Heath JK, Rodan GA (1989) Estradiol enhances insulin-like growth factor I mRNA and has anabolic effects in osteoblastic cells from rat calvaria and long bones. $J$ Bone $\mathrm{Min}$ Res 4 (supplt 1), S256

Faugère MC, Okamoto $S$, De Luca HF, Malluche $\mathrm{HH}$ (1986) Calcitriol corrects bone loss induced by oophorectomy in rats. Am J Physiol 250, E35-E38

Franchimont $P(1989)$ Effect of repetitive administration of growth hormone-releasing hormone on growth hormone secretion, insulinlike growth factor 1 and bone metabolism in postmenopausal women. Acta Endocrinol 120, 121-128
Frost HM (1969) Tetracycline-based histological analysis of bone remodeling. Calcif Tissue Res 3, 211-239

Frost HM (1973) Bone remodeling and its relationship to metabolic bone disease. Thomas CC, Springfield, 3-27

Frost HM (1985) The pathomechanics of osteoporosis. Clin Orthop Rel Res 200, 198-225

Garn SM, Rohman CG, Wagner B (1967) Bone loss as a general phenomenon in man. Fed Proc 26, 1729-1736

Gilsanz J, Roe TF, Antunes J, Carlson M, Duarte ML, Goodman WG (1991) Effect of dietary calcium on bone density in growing rabbits. Am J Physiol 260, E471-E476

Gong JK, Arnold JS, Cohn SH (1964) Composition of trabecular and cortical bone. Anat Record 149, 325-331, 1964

Hansard SL, Crowder HM (1957) The physiological behavior of calcium in the rat. $J$ Nutr 62 , 325-339

Heaney RP (1982) Calcium intake requirement and bone mass in the elderly. $J$ Lab Clin Med $100,309-312$

Heaney RP, Recker RR, Saville PD (1978) Menopausal changes in bone remodeling. $J$ Lab Clin Med 92, 964-970

Hock JM, Gera I, Fonseca J, Raisz LG (1988) Human parathyroid hormone (1-34) increases bone mass in ovariectomized and orchidectomized rats. Endocrinology 122, 2899-2904

Hock JM, Wood RJ (1991) Bone response to parathyroid hormone in aged rats. Cells Materials Supplt 1, 53-58

Hodgen GD, Goodman AL, O'Connor A, Johnson DK (1977) Menopause in rhesus monkeys: model for study of disorders in the human climacteric. Am J Obstet Gynecol 127 , 581-584

Hodgkinson A, Aaron JE, Horsman A, Mc Lachlan MSF, Nordin BEC (1978) Effect of oophorectomy and calcium deprivation on bone mass in the rat. Clin Sci Mol Med 54, 439-446

Holliday R (1992) The ancient origins and causes of ageing. News Physiol Sci 7, 38-40

Hori M, Uzawa T, Morita K, Noda T, Takahashi H, Inone J (1988) Effect of human parathyroid hormone (1-34) on experimental osteopenia in rats induced by ovariectomy. Bone $\operatorname{Min} 3,193$ 199 
Horst RL, Goff JP, Reinhardt TA (1990) Advancing age results in reduction of intestinal and bone 1,25-dihydroxyvitamin $D$ receptor. Endocrinology 126, 1053-1058

Ibbotson KJ, Orcutt CM, D'Souza SM, Paddock $\mathrm{CL}$, Arthur JA, Jaukowsky ML, Boyce RW (1992) Contrasting effects of parathyroid hormone and insulin-like growth factor I in an aged ovariectomized rat model of postmenopausal osteoporosis. J Bone Min Res 7, 425432

Izawa $Y$, MakitaT, Ichiki $\mathrm{H}$ (1985) Effect of active vitamine $D_{3}$ analogs on the osteoporosis of rats caused by ovariectomy. Japan J Vet Sci 47, 349-356

Jérome CP, Kimmel DB, McAlister JA, Weaver DS (1986) Effects of ovariectomy on iliac trabecular bone in baboons (Papio anubis). $\mathrm{Cal}$ cif Tissue Int 39, 206-208

Jilka RL, Hangoc C, Girasole G, Passeri G, Williams DC, Abrams JS, Boyce B, Broxmeyer $H$, Manolagas SC (1992) Increased osteoblast development after estrogen loss: mediation by interleukin-6. Science 257, 88-91

Johnston CC, Hiu SL, Witt RM, Appledorn R, Baker RS, Longcope C (1985) Early menopausal changes in bone mass and sex steroids. J Clin Endocrinol Metab 61, 905-911

Johnston CC, Miller JZ, Slemenda CW, Reister TK, Hui S, Christian JC, Peacock M (1992) Calcium supplementation and increases in bone density in children. N Engl J Med 327 , 82-87

Johnston CC, Slemenda CW (1992) Changes in skeletal tissue during the aging process. Nutr Rev 50, 385-387

Joss EE, Sobel EM, Zuppinger KA (1963) Skeletal maturation in rats with special reference to order and time of epiphyseal closure. Endocrinology 75, 117-122

Juhn A, Weiss A Mendes D, Silbermann M (1991) Non-invasive assessment of bone mineral density during maturation and aging of wistar female rats. Cells and Materials supplt 1: 1924

Kalu DN (1984) Evaluation of pathogenesis of skeletal changes in ovariectomized rats. Endocrinology 115, 507-512

Kalu DN (1991) The ovariectomized rat model of postmenopausal bone loss. Bone Min 15, 175192
Kalu DN, Hardin RR, Cockerham R, Yu BP (1988) Aging and dietary modulation of the rat skeleton and parathyroid hormone. Endocrinology 115, 1239-1247

Kalu DN, Liu CC, Hardin RR, Hollis BW (1989) The aged rat model of ovarian hormone deficiency bone loss. Endocrinology 124, 7-16

Karambolova KK, Snow GR, Anderson C (1987) Effects of continuous $17 \beta$-estradiol administration on the periosteal and corticoendosteal envelope activity in spayed Beagles. Calcif Tissue Int 40, 12-15

Kawase M, Tsuda M, Matsuo T (1989) Accelerated bone resorption in senescence-accelerated mouse (SAM-P/6). J Bone Min Res 4, 359-364

Kiebzack GM, Smith R, Gundberg CC, Hove JC, Sacktor B (1988) Bone status of senescent male rats: chemical, morphometric and mechanical analysis. J Bone Min Res 3, 3745

Kimmel DB (1991a) The oophorectomized Beagle as an experimental model for estrogen-depletion bone loss in the adult human. Cells and Materials supplt 1, 75-84

Kimmel DB (1991b) Quantitative histologic changes in the proximal tibial growth cartilage of aged female rats. Cell and Materials supplt 1, 11-18

Kimmel DB, Jee WSS (1982) A quantitative histologic study of bone turnover in young adult Beagles. Anat Record 203, 35-51

Kimmel DB, Wronski TJ (1990) Non-destructive measurement of bone mineral in femurs from ovariectomized rats. Calcif Tissue Int 46 : 101110

Komm BS, Terpening CM, Benz DV, Graeme KA, Gallegos A, Kore M, Greene GL, O'Malley BW, Haussler MR (1988) Estrogen binding, receptor $\mathrm{mRNA}$ and biologic response in osteoblast-like osteosarcoma cells. Science 241, 81-84

Lazowski DA, Anderson C, Fraher LJ (1991) Dietary calcium supplementation and preservation of bone mass in oophorectomized rats. Cells and Materials supplt 1, 119-124

Li XJ, Jee WSS, Ke HZ, Mori S, Akamine T (1991) Age-related changes of cancellous and cortical bone histomorphometry in female Sprague-Dawley rats. Cells and Materials supplt 1, 25-35 
Lindgren JU, Lindholm TS (1979) Effect of 1. alpha-hydroxyvitamin $D_{3}$ on osteoporosis in rats induced by oophorectomy. Calcif Tissue Int 27, 161-164

Longscope C, Hoberg L, Sleuterman S, Barin D (1989) The effect of ovariectomy on spine bone mineral density in rhesus monkeys. Bone 10, 341-344

Lundon KL, Grynpas M (1993) The long-term effect of ovariectomy on the quality and quantity of cortical bone in the young Cynomolgus monkey: comparison of density fractionation and histomorphometric techniques. Bone 14, 389-395

Malluche $\mathrm{HH}$, Faugère MC (1990) Role of calcitriol in the management of osteoporosis. Metabolism 39 ( 4 supplt 1 ), 24-26

Malluche $\mathrm{HH}$, Faugère $\mathrm{MC}$, Rush $\mathrm{M}$, Friedler $\mathrm{R}$ (1986) Osteoblastic insufficiency is responsible for maintenance of osteopenia after loss of ovarian function in experimental Beagle dogs. Endocrinology 122, 1998-2005

Mann DR, Gould KG, Collins DC (1990) A potantial primate model for bone loss resulting from medical oophorectomy or menopause. J Lab Clin Med 71, 105-110

Martin RB, Butcher RL, Sherwood LL, Buckendahl P, Boyd RD, Farris D, Sharkey N, Dannucci $G$ (1987) Effects of ovariectomy in Beagle dogs. Bone 8, 23-21

Matkovic V (1992) Influence of nutritional factors on peak bone mass. In: Ostéoropose : pour une prévention nutritionnelle du risque $(B$ Lesourd, CH Rapin, P Sachet, eds). Cerin, Paris, 71-84

Mbuyi-Muamba JM, Dequecker J (1986) Bone mass measured by photon absorptiometry and radiogravimetry in Wistar rats. Invest Radiol 21, 336-339

Miller LC, Weaver DS, Mc Alister JA, Koritvik DR (1986) Effects of ovariectomy on vertebral trabecular bone in the Cynomolgus monkey (Macaca Fascicularis). Calcif Tissue Int 38, 62-67

Mosekilde L, Kragstrup J, Richards A (1987) Compressive strength, ash weight, and volume of vertebral trabecular bone in experimental fluorosis in pigs. Calcif Tissue Int 40, 318-322

Mosekilde L, Weisbrode SE, Safron JA et al (1993) Calcium-restricted ovariectomized Sinclair S-1 minipigs: an animal model of osteopenia and trabecular plate perforation. Bone $14,379-382$
Nishunoto SK, Chang $\mathrm{CH}$, Gendler E, Stryker WF, Nimni N (1985) The effect of aging on bone formation in rats: biochemical and histological evidence for decreased bone formation capacity. Calcif Tissue Int 37 : 617-624

Nordin BEC, Need AG, Morris HA, Horowitz M (1985) New approaches to the problems of osteoporosis. Clin Orthop Relat Res 200, 181 197

Ott SM (1990) Editorial: attainment of peak bone mass. J Clin Endocrinol Metab 71, 1082A$1082 \mathrm{C}$

Parfitt AM, Mathews CHE, Villanueva AR, Kleerekoper M, Frame B, Rao DS (1983) Relationships between surface, volume and thickness of iliac trabecular bone in aging and osteoporosis. Implication for the microanatomic and cellular mechanisms of bone loss. $J$ Clin Invest 72, 1396-1409

Parks NJ, Jee WSS, Dell RB, Miller GE (1986) Assessment of cortical and trabecular bone distribution in the Beagle skeleton by neutron activation analysis. Anat Record 215, 230250

Pastoureau P, Arlot ME, Caulin F, Barlet JP, Meunier PJ, Delmas PD (1989) Effects of oophorectomy on biochemical and histological indices of bone turnover in ewes. J Bone Min Res 4 (supplt 1), S237

Pocock NA, Eisman JA, Hopper JL, Yeates MG, Sambrook PN, Eberl S (1987) Genetic determinants of bone mass in adults: a twin study. $J$ Clin Invest 80, 706-710

Pohiman RL, Darby LA, Lechner AJ (1985) Morphometry and calcium contents in appendicular and axial bones of exercised ovariectomized rats. Am J Physiol 248, R12-R17

Pope NS, Gould KG, Anderson DC, Mann DR (1989) Effects of age and sex on bone density in the rhesus monkey. Bone 10, 109-112

Pouilles JM, Ribot C (1992) Choix et validité des méthodes de mesure de la masse osseuse : applications aux études nutritionnelles. In : Ostéoropose : pour une prévention nutritionnelle du risque (B Lesourd, $\mathrm{CH}$ Rapin, $\mathrm{P}$ Sachet, eds). Cerin, Paris, 13-21

Prior JC (1990) Progesterone as a bone trophic hormone. Endocr Rev 11, 386-398

Raab DM, Chenshaw TD, Kimmel DB, Smith EL (1991) A histomorphometric study of cortical bone activity during increased weight-bearing exercise. J Bone Min Res 6, 741-749 
Reeve J, Meunier PJ, Parsons JA et al (1980) Anabolic effect of human parathyroid hormone fragment on trabecular bone in involutional osteoporosis; a multicenter trial. Brit J Med $280,1340-1344$

Reid IR, Ames RW, Evans MC, Gamble GD, Sharpe SJ (1993) Effect of calcium supplementation on bone loss in postmenopausal women. N Engl J Med 328, 460-464

Reisenfeld R (1981) Age changes of bone life and mass in two strains of senescent rats. Acta Anat 109, 64-69

Renier JC, Audran M (1989) L'os et le tissu osseux au cours de la vie. In: Le tissu osseux ( $L$ Teot, J Vidal, J Dossa, eds). Sauramps Medical, Montpellier, 141-162

Riggs BL, Melton LJ (1986) Involutional osteoporosis. NEngl J Med 26, 1676-1684

Riggs BL, Wahner HW, Melton LJ, Richelson LS, Judd HL, O'Fallon WM (1987) Dietary calcium intake and rates of bone loss in women. $J$ Clin Invest 80, 973-982

Riis BJ, Rodbro P, Christiansen C (1986) The role of serum concentrations of sex steroids and bone turnover in the development and occurrence of postmenopausal osteoporosis. Calcif Tissue int 38, 318-322

Rodgers JB, Monier-Faugère MC, Malluche $\mathrm{H}$ (1993) Animal models for the study of bone loss after cessation of ovarian function. Bone $14,369-377$

Saville PD (1969) Changes in skeletal mass and fragility with castration in the rat: a model of osteoporosis. J Am Geriat Soc 17, 155-164

Schapira D, Lotan-Miller R, Barzilai D, Silbermann $M$ (1991) The rat as model for studies of the aging skeleton. Cells and Materials supplt 1, 181-188

Schnitzler CM, Ripamonti U, Mesquita JM (1993) Bone histomorphometry in baboons in captivity. Bone 14, 383-387

Schot LPC, Schuurs AHWM (1990) Sex steroids and ostoporosis. J Steroid Molec Biol 37, 167182

Shen V, Dempster DW, Mellish TWE, Birchman R, Horbert W, Lindsay R (1992) Effects of combined and separate administration of low dose human parathyroid fragment (1-34) and $17 \beta$-estradiol on bone histomorphometry in ovariectomized rats with established osteopenia. Calcif Tissue int 50, 214-220
Silbermann M, Safari M, Schapira D, Leichter M, Steinberg R (1989) Structural and compositional changes in aging bone: osteopenia in lumbar vertebrae of wistar female rats. Scanning Microsc 3, 945-952

Simon MR (1984) The rat as an animal model for the study of senile idiopathic osteoporosis. Acta Anat 119, 248-250

Stepan JJ, Pospichal J, Presl J, Pacovsky V (1987) Bone loss and biochemical indices of bone remodeling in surgically induced postmenopausal women. Bone 8, 279-284

Tabuchi C, Simmons DJ, Fausto A, Russell JE, Binderman I, Avioli LV (1986) Bone deficit in ovariectomized rats: functional contribution of the marrow stromal cell population and the effect of oral dihydrotachysterol treatment. $J$ Clin Invest 78, 637-642

Takano-Yamamoto T, Rodan GA (1990) Direct effects of $17 \beta$-estradiol on trabecular bone in ovariectomized rats. Proc Natl Acad Sci USA 87, 2172-2176

Thompson DD, Seedor JG, Quartuccio $H$ et al (1992) The bisphosphonate, alendronate, prevents bone loss in ovariectomized baboons. J Bone Miner Res 7, 951-960

Tommerup LV, Raab DM, Crenshaw TD, Smith EL (1993) Does weight exercise affect nonweight bearing bone? J Bone Min Res 9, 1053-1058

Trotter M, Hixon BB (1974) Sequantial changes in weight, density, and percentage ash weight of human skeletons from an early fetal period through old age. Anat Record 179, 1-18

Tsuboyama T, Takahashi K, Yamamuro T, Hosokawa M, Takeda T (1993) Cross-mating study on bone mass in the spontaneously osteoporotic mouse (SAM-P/6). Bone Min 23, 57-64

Turner RT, Vandersteenhoven JJ, Bell NH (1987) The effects of ovariectomy and $17 \beta$-estradiol on cortical bone histomorphometry in growing rats. J Bone Min Res 2, 115-122

Vanderschueren $D$, Vanherck $E$, Schot $P$, Rush $E$, Einhorn T, Geusens P, Bouillon R (1993) The aged male rat as a model for human osteoporosis: evaluation by non-destructive measurements and biomechanical testing. Calcif Tissue Int 53, 342-346

Wei LL, Leach MW, Miner RS, Dewers LM (1993) Evidence for progesterone receptors in human osteoblast-like cells. Biochem Biophys Res Commun 195, 525-532 
Wronski TJ, Lowry PL, Walsh CC, Ignaszewski LA (1985) Skeletal alterations in ovariectomized rats. Calcif Tissue Int 37, 324-328

Wronski TJ, Schenck PA, Walsh CC (1986a) Obesity partially protects against osteopenia in ovariectomized rats (Abstract). J Bone Miner Res 1 (supplt 1), 251

Wronski TJ, Walsh CC, Ignaszewski LA (1986b) Histologic evidence for osteopenia and increased bone turnover in ovariectomized rats. Calcif Tissue Int 43, 179-183

Wronski TJ, Cintron M, Dann LM (1988a) Temporal relationship between bone loss and increased bone turnover in ovariectomized rats. Calcif Tissue Int 43, 179-183

Wronski TJ, Cintron M, Doherty AL, Dann LM (1988b) Estrogen prevents osteopenia and depresses bone turnover in ovariectomized rats. Endocrinology 123, 681-686
Wronski TJ, Dann LM, Horner SL (1989a) Time course of vertebral osteopenia in ovariectomized rats. Bone 10, 295-301

Wronski TJ, Dann LM, Scott KS, Croobe LR (1989b) Endocrine and pharmacological suppressors of bone turnover protect against osteopenia in ovariectomized rats. Endocrinology 125, 810-816

Wronski TJ, Dann LM, Scott KS, Cintron M (1989c) Long-term effects of ovariectomy and aging on the rat skeleton. Calcif Tissue Int 45, 360-366

Wronsky TJ, Yen CF (1991) The ovariectomized rats as an animal model for postmenopausal bone loss. Cells and Materials supplt 1, 69-74

Yeh JK, Lin CC, Aloia JF, Foto A (1991) Effect of treadmill exercise and ovariectomy on femoral and lumbar vertebrae in young and adult rats. Cells and Materials supplt 1, 159-166 